\title{
Theoretical Study of the Influence of Chemical Defects on the Molecular Properties of Semiconducting Polymers
}

\author{
Marta M. D. Ramos ${ }^{1,2, a}$, Helena M. G. Correia ${ }^{2, b}$ and \\ Hélder M. C. Barbosa ${ }^{1,2, c}$ \\ ${ }^{1}$ Department of Physics, University of Minho, Campus de Gualtar, 4710-057 Braga, Portugal \\ ${ }^{2}$ Centre of Physics, University of Minho, Campus de Gualtar, 4710-057 Braga, Portugal

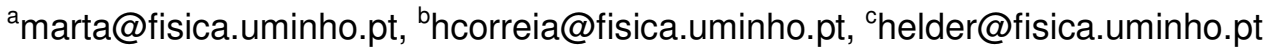

Keywords: Atomistic modelling, PPV, Chemical defects, Electronic properties

\begin{abstract}
Semiconductor polymers are successfully implemented in a broad range of applications such as light emitting diodes, field effect transistors and photovoltaic devices. Most of the achievements reached in the development of these devices were obtained at experimental level, being difficult to indentify individually the influence of each factor that limits and controls these devices efficiency. One of the factors that strongly influence the performance of polymer-based devices is the presence of chemical defects in the polymer strands that change their molecular properties. As a result, these polymer strands can work like traps or deep energetic states for charge transport, leading, for instance, to a decrease on charge mobility. At experimental level it is a difficult task to isolate the influence of each type of chemical defects individually on the molecular properties of the polymer strands. It is in this context that theoretical modelling seems to be the most suitable approach to get a deep understanding of the influence of chemical defects on the molecular properties of semiconductor polymers. By performing quantum molecular dynamics calculations we study the influence of chemical defects on the molecular properties of poly(paraphenylenevinylene) (PPV). Our results show clearly a significant difference on the electronic properties of defective polymer strands (e.g. charge carrier localization, ionization potential, electron affinity and electric-field threshold for charge carrier mobility along the polymer backbone) as compared with defect-free strands.
\end{abstract}

\section{Introduction}

Conjugated polymers exhibiting semiconductor behaviour are being extensively investigated as an alternative to inorganic semiconductors for electronic and optoelectronic applications [1]. In these organic materials the conformational disorder of the polymer chains during the solution processing or the interruption of conjugation during the polymerization process produces chains of linked conjugated segments with variable lengths and terminations [2]. The electronic coupling dictates how the injected charge is transport among the conjugated segments. Two basic types of charge transport can occur: charge migration along the conjugated segment backbone and charge hopping between segments in close proximity [3].

Dynamic model, that includes both charge transport mechanisms, aimed at describing the effects of molecular properties and the spatial arrangements of the conjugated segments within the polymer layer on charge transport properties of conjugated polymers was proposed recently [4]. So far this model has been just applied to pristine polymer layers with no chemical defects, which can only be deposited under very restrictive conditions as compared with real conditions of those materials in the devices.

The difficulty in developing this model to account the several experimental observations in conjugated polymers is largely due to the lack of experimental data concerning the nature of the 
chemical defects and their effects on charge transport processes described above, because most of the available measurements rely on the techniques averaging their effects in space. Molecular modelling can have a huge added value here, since it allows one to compute the effects of chemical defects in a given conjugated polymer strand as regard the energies involved in adding or removing electrons from the strand, the nature and localization of the charge-induced carriers on the polymer backbone and their mobility along the conjugated system. Without such knowledge carrier transport processes in conjugated polymers cannot be well understood, which motivated the present theoretical study. Because poly(para-phenylenevinylene) (PPV) is both important in its applications and simple in its chemical structure, we have chosen it as a model system to address these questions that should also apply to other conjugated polymers.

\section{Theoretical Methods}

Since in conjugated polymers there is a strong connection between the electronic and geometric structures, it is necessary to perform simultaneous self-consistent calculations of electronic structure and geometry relaxation in order to study the influence of chemical defects on electronic properties of an isolated PPV strand.

In this work we use the theoretical approach implemented in the CHEMOS code [5]. The electronic structure calculations were performed by a semi-empirical application of the HartreeFock Theory called CNDO (Complete Neglect of Differential Overlap). The parameters selected for our calculations were the ones that give the best overall fit to minimal basis set Ab Initio HartreeFock calculations for small molecules, a linear combination of atomic orbitals and a cluster model framework. The gradients of the self-consistent internal energy, evaluated at the given atom positions, yield the inter-atomic forces. A molecular dynamics method is used in parallel to move the atoms according to those forces. In these simulations the atoms of an initial guess geometric structure move, within the Born-Oppenheimer approximation, on the potential energy surface obtained directly from the electronic structure calculation until the equilibrium geometry is reached.

\section{Results and Discussion}

The ground state geometries and Mulliken atomic charge distribution of defect-free uncharged PPV strands were found by examining straight planar molecules of $\mathrm{C}_{8 \mathrm{n}} \mathrm{H}_{6 \mathrm{n}+2}$, where $\mathrm{n}$ is the number of repeat units. PPV strands of different lengths were obtained by varying the number of repeat units from 2 to 11. Three different chemical defects were considered in this work: a vinyl termination end, the saturation of a vinyl group with hydrogen, the formation of a non-aromatic carbonyl group. Fig. 1 shows the position of those chemical defects for a PPV strand of seven repeat units long.

(a)

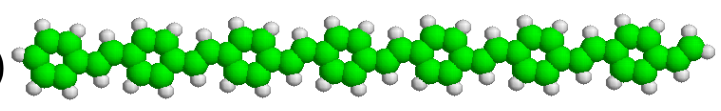

(b)

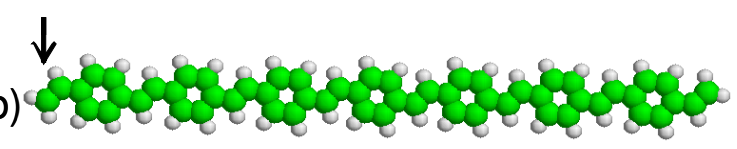

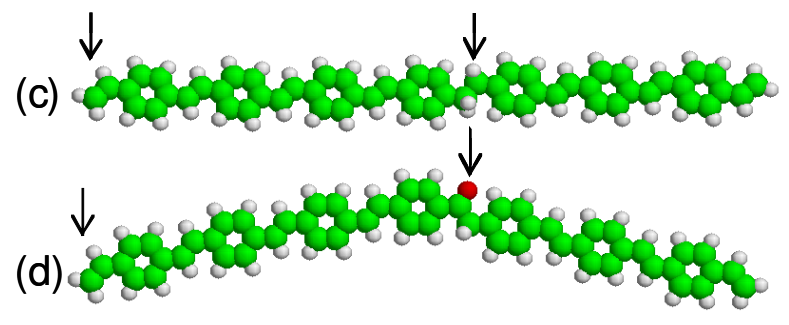

Fig. 1 - The ground state geometry of uncharged PPV strands with 7 repeat units long and the following chemical defects: (a) defect-free; (b) a vinyl termination end on the left-hand side; (c) a vinyl termination end on the left-hand side and the saturation of a vinyl group with hydrogen; (d) a vinyl termination end on the left-hand side and the formation of a non-aromatic carbonyl group. The arrows indicate the position of the defects.

When an electron is added or removed from the PPV strands with and without chemical defects, a charge rearrangement among the PPV atoms is induced. Fig. 2 shows the charge storage on carbon and hydrogen atoms along the polymer strands depicted in Fig. 1. 
(a)
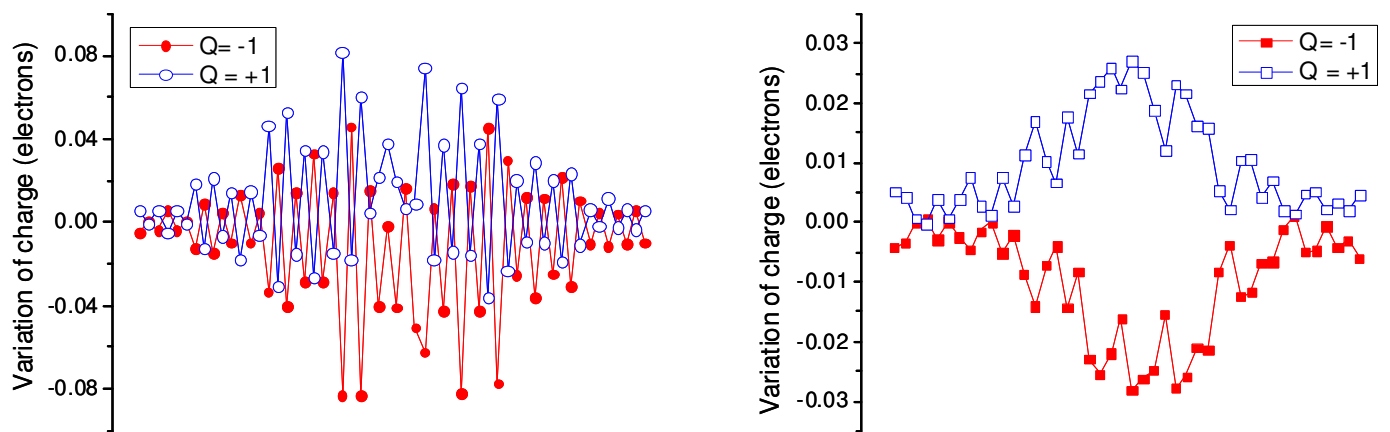

(b)
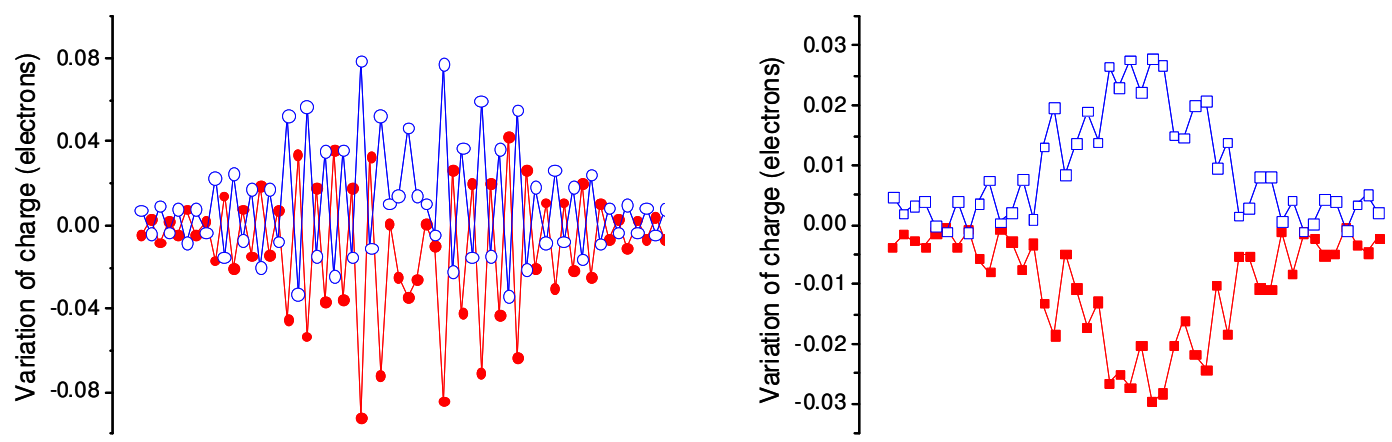

(c)
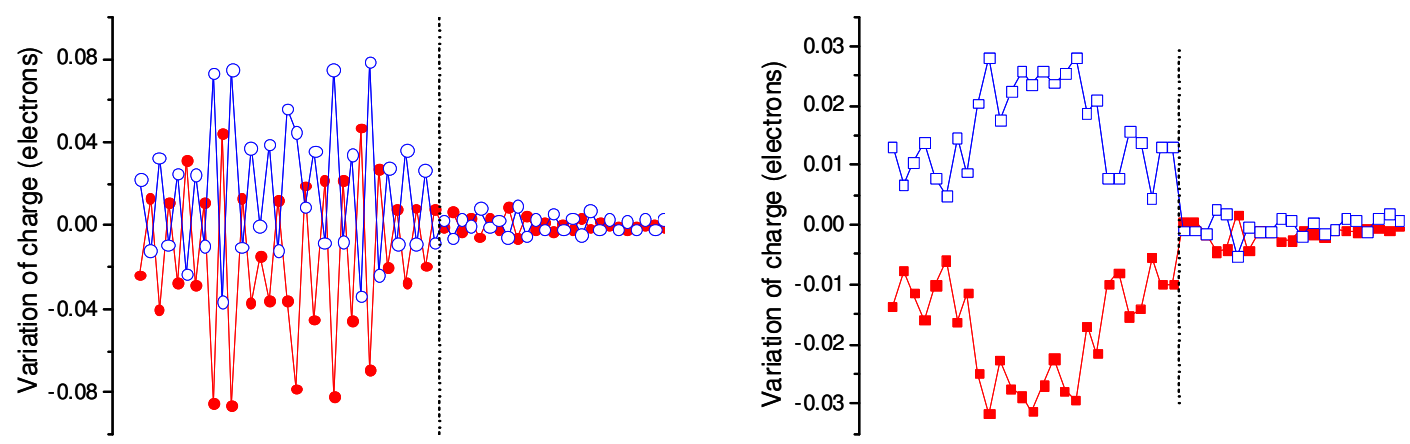

(d)
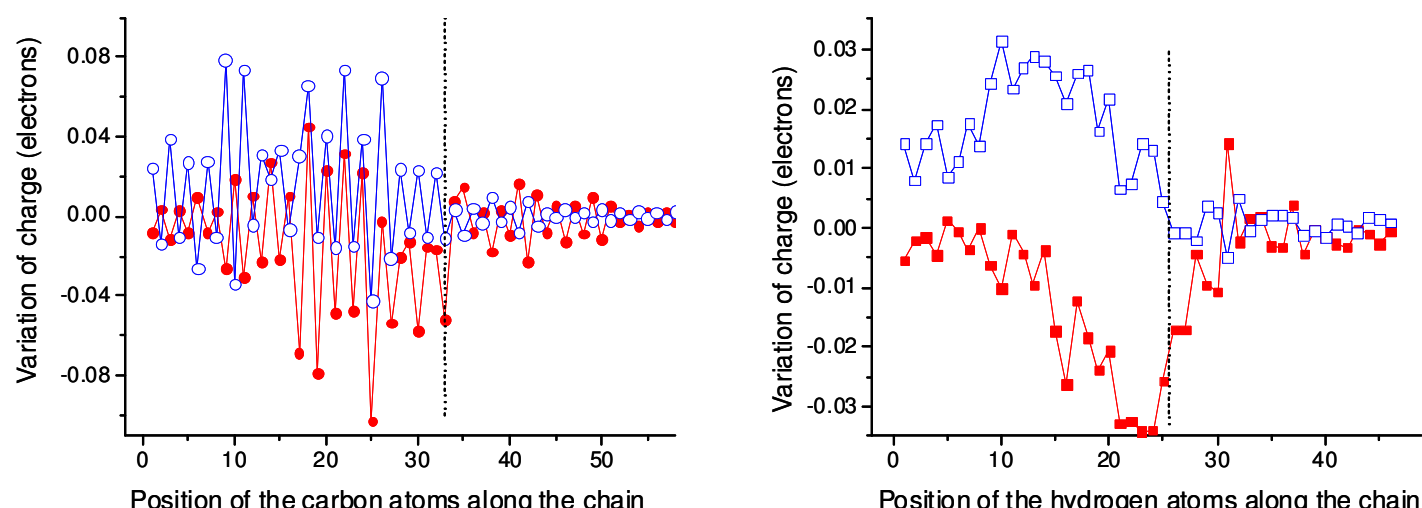

Fig. 2 - Changes in Mulliken charges for carbon (left-hand side) and hydrogen (right-hand side) atoms of the strands depicted in Fig. 1 when an electron is added $(\mathrm{Q}=-1)$ (filled marks) or removed $(\mathrm{Q}=+1)$ (empty marks) from them. The vertical broken lines indicate the position of the in-chain chemical defects. The solid lines are a guide to the eye.

The injected charge is localized on carbon and hydrogen atoms of the polymer backbone at the central region of the polymer strand, when the conjugation along the strand is not lost, or within the longest conjugated subunit formed, when the conjugation between two phenyl groups is lost, and 
extends over a few nanometres. For both positive and negative charged PPV strands with and without chemical defects, our results suggest an alternation of the charge sign on adjacent atoms only for carbons. However, different charge distribution profiles are obtained depending on the type of defect. The in-chain imperfections containing carbonyl or saturated bonds affect mainly the localization of the injected charge and the charge distribution profile in less extended.

The injection of charge described above is accompanied by a charge-induced geometry relaxation extending over the same distance as the charge storage. The extent of the distortion can be obtained by calculating the change in the difference between adjacent carbon-carbon bond lengths along the strand from their ground state uncharged values (i.e. variation of dimerisation). The distortion patterns for PPV strands of 7 repeat units long with and without the chemical defects considered in this work are shown in Fig. 3. Although the width of the geometric distortion in charged PPV strands is rather similar for strands with and without chemical defects, the chemical defects induce different amplitudes and shapes, the effect being more pronounced for strands containing carbonyl or saturated bonds.
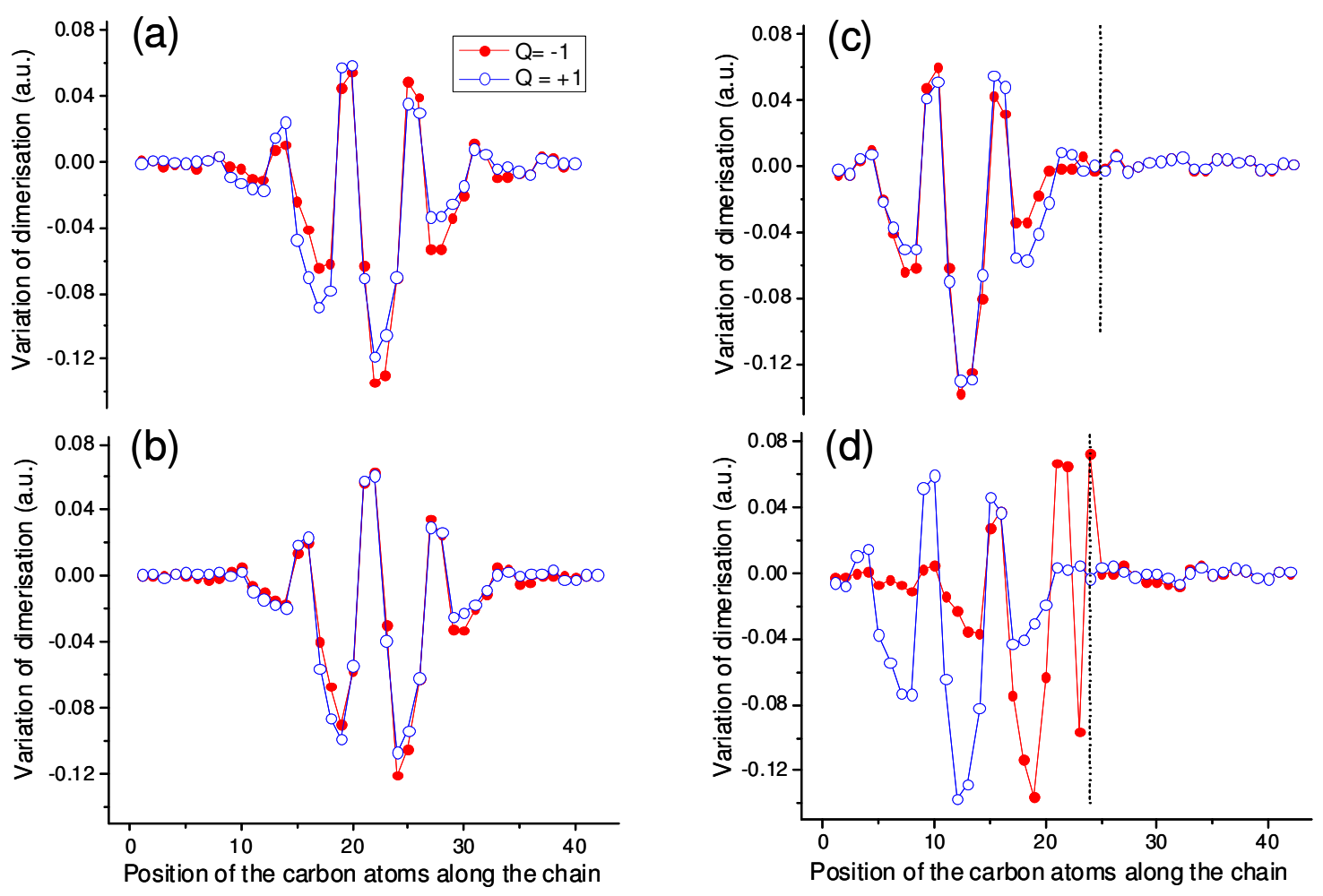

Fig. 3 - Change in the difference between adjacent carbon-carbon bond lengths along the backbone caused by adding or removing one electron from the PPV the strands depicted in Fig. 1. The marks indicate the data points that were calculated explicitly, whilst the curves are simply a guide to the eye. The vertical broken lines indicate the position of the in-chain imperfections.

Since electron affinity (ionization potential) differences between conjugated segments of different length drive electron (hole) transfer within the polymer network, we must consider the effect of the chemical defects on the electron affinity (ionization potential) of the PPV strands as a function of their length (measured in the number of monomer units). The ionization potential (IP) (the energy needed to remove an electron from the neutral system) and the electron affinity (EA) (the energy gained on adding an electron to the neutral system), are calculated from the energy differences between the optimized geometry of neutral and charged systems and given by: 


$$
\begin{aligned}
& \mathrm{IP}=\mathrm{E}_{\mathrm{Q}=+1}-\mathrm{E}_{\mathrm{Q}=0} \\
& \mathrm{EA}=\mathrm{E}_{\mathrm{Q}=0}-\mathrm{E}_{\mathrm{Q}=-1}
\end{aligned}
$$

where $E_{Q}$ is the internal energy of the system with optimized geometry and total charge Q. Fig. 4 shows the IP and EA calculated for the PPV strands with the chemical defects depicted in Fig.1.
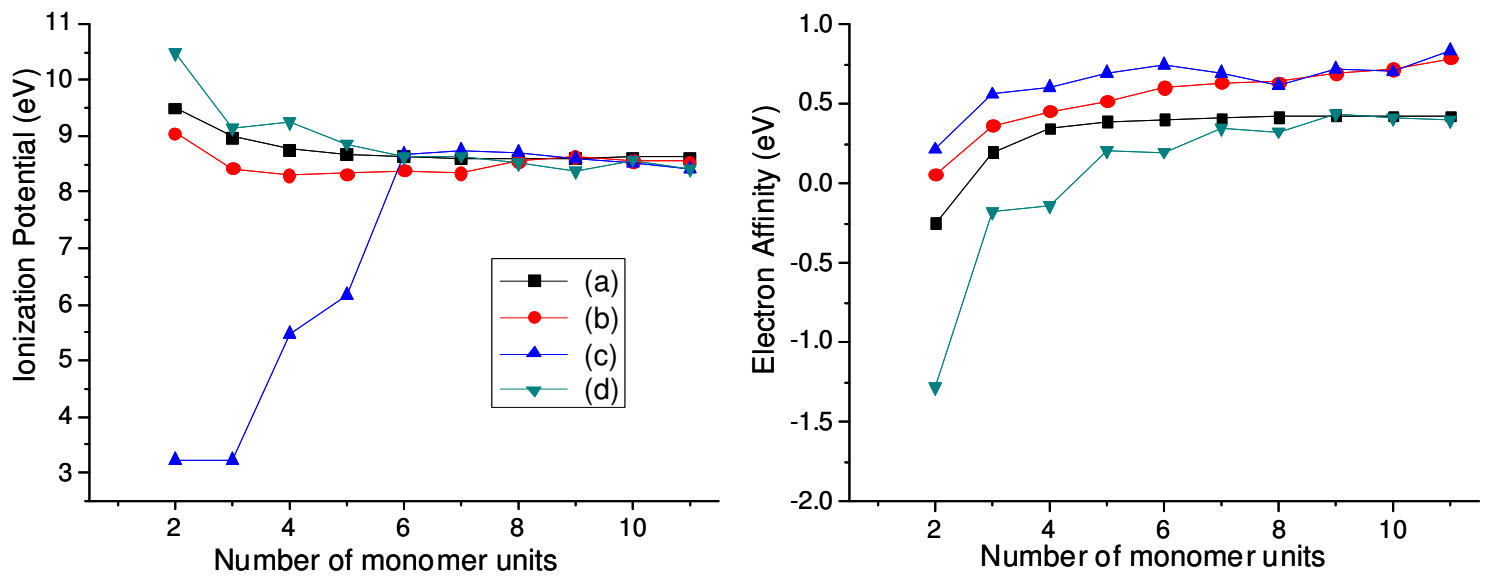

Fig. 4 - The ionization potential (left-hand side) and the electron affinity (right-hand side) of PPV strands with variable length (measured in the number of monomer units) and the following chemical defects: (a) defect-free (squares); (b) a vinyl termination end (circles); (c) a vinyl termination end and the formation of a non-aromatic carbonyl group (triangles); (d) a vinyl termination end and the saturation of a vinyl group with hydrogen (inverted triangles). The lines are just a guide to the eye.

The PPV strands, with and without chemical defects, have positive ionization potentials and electron affinities. Since a positive difference between the electron affinity of the strand considered with and without the chemical defect signifies a potential trap for the electron and conversely for the hole, our results suggest that chemical defects linked with the presence of a vinyl termination end and a carbonyl group that are not part of the repeat unit that constitutes the backbone of the conjugated strand may act as electron traps whereas the presence of a vinyl termination end and the saturation of the vinyl group with hydrogen is predicted to produce a trap for holes. Among the chemical defects considered in this work, the one containing a carbonyl group is predicted to be the deepest electron trap and the magnitude of the trap is less dependent on the strand length. The ionization potentials have also been calculated for these chemical defects in order to estimate their potential in the injection of positive charge from the anode. The significant decrease in the ionization potential of short PPV strands induced by the presence of oxygen-related defect can be a plausible explanation for PPV be considered a good hole transport material [6], with an unbalance of positive and negative carrier currents, since the low ionization potential of the shorter conjugated segments should lead to low hole injection barriers from the electrode.

Although the presence of oxygen-related defect can act as trap for holes and electrons, its presence increases EA in all PPV strands as compared with the defect-free strands independently of their length while for holes the presence of the carbonyl group just affects the short PPV-based strands. Since in a PPV network the length of the conjugated segments can vary from 2 to 11 , this means that the presence of oxygen-related defect affects more the transport of electrons than holes.

In order to assess the influence of the chemical defects on the mobility of charge (electron or hole) carriers, we have focused our attention on their motion towards the right-hand side of the polymer strands depicted in Fig. 1. For electric fields lower than the value shown in Table 1, no charge mobility was predicted. When the applied field is above this threshold value, the injected 
charge and the lattice distortion move together along the PPV strand until the strand end or the inchain imperfection, i.e. carbonyl or saturated bonds, is reached. For strands with in-chain imperfections the electric field thresholds for electron and hole mobility is higher than for defectfree strand and the injected charge can only cross the in-chain imperfections by breaking the polaron-like carrier for fields much higher than those occurring in the devices operation. The vinyl termination group leads to a decrease in the electric field threshold for hole mobility along the strand.

\begin{tabular}{|l|c|c|}
\hline PPV strand with: & $\begin{array}{l}\text { Electric field threshold for } \\
\text { electron mobility [MV/cm] }\end{array}$ & $\begin{array}{l}\text { Electric field threshold for } \\
\text { hole mobility [MV/cm] }\end{array}$ \\
\hline defect-free & 3.105 & 2.644 \\
\hline vinyl termination end & 3.258 & 2.290 \\
\hline $\begin{array}{l}\text { vinyl termination end and } \\
\text { saturation of a vinyl group }\end{array}$ & 3.163 & 5.092 \\
\hline $\begin{array}{l}\text { vinyl termination end and a non- } \\
\text { aromatic carbonyl group }\end{array}$ & 8.190 & 4.810 \\
\hline
\end{tabular}

Table 1 - The electric field threshold for charge carrier mobility along the polymer backbone for PPV strands of 7 repeat units long with and without chemical defects.

\section{Conclusions}

In this work we have studied the influence of the chemical defects on the static and dynamic molecular properties of PPV strands concerning the injection of electrons and holes and the mobility of the formed charge carriers along the strand. Our results suggest that chemical defects do not change the nature of the charge carriers but mainly their localization. Among the chemical defects considered in this work, the vinyl termination group and the carbonyl bond are predicted to be electron traps whereas the saturation of the vinyl group is predicted to be hole trap. The electric field threshold for charge mobility along PPV strands with chemical defects is quite different from the defect-free one due to the different electron donor and acceptor capabilities of the chemical defects. The results reported here are a useful data for further developing of nanoscale modelling of polymer-based electronic devices, which is currently impossible to obtain experimentally.

\section{Acknowledgement}

We are indebted to the Portuguese Foundation for Science and Technology (FCT) for financial support under the contract CONC-REEQ/443/EEI/2005 and the PhD grand SFRH/BD/22143/2005.

\section{References}

[1] N. Tesseler, N. T. Harrison and R. H. Friend, Adv. Mat. Vol. 10 (1998) p. 64

[2] E. Collini and G. E. Scholes: Science Vol. 263 (2009), p. 369

[3] M. M. D. Ramos and H. M. G. Correia: J. Phys.-Condes Matter Vol. 18 (2006) p.S429

[4] H. M. C. Barbosa and Marta M. D. Ramos: Mater. Sci. Eng. B (2009), in press DOI 10.1016/j.mseb.2008.09.031

[5] D. S. Wallace, A. M. Stoneham, W. Hayes, A. J. Fisher and A. H. Harker: J. Phys.-Condes Matter Vol. 3 (1991) p. 3879

[6] H. C. F. Martens, J. N. Huiberts and P. W. M. Blom: Appl. Phys. Lett.: Vol. 77 (2000) p.1852 